\title{
Acoustic evaluation of modular greenery noise barriers
}

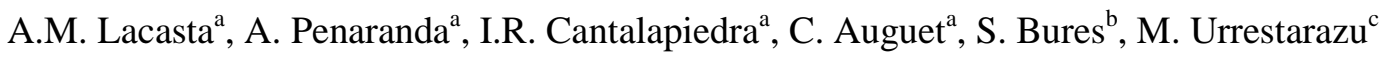 \\ ${ }^{a}$ Departament de Física, EPSEB, Universitat Politècnica de Catalunya, 08028 Barcelona, \\ Spain \\ ${ }^{b}$ Buresinnova S. A., Barcelona, Spain \\ ${ }^{c}$ Departamento de Agronomía, Universidad de Almería, Almería 04120, Spain
}

\begin{abstract}
Green noise barriers have become an alternative means of reducing urban traffic noise. In this paper, the acoustic performance of a modular greenery noise barrier is evaluated. In situ measurements of noise reflection were performed using an experimental prototype to estimate the sound absorption coefficients. These coefficients were found to have values of approximately 0.7 , higher than those previously found in laboratory measurements for a similar system with a lower vegetation density. The obtained values were input into software for predicting environmental noise to analyse the expected performance of such barriers, particularly in the case of a pair of parallel barriers. A comparison with the results for reflective barriers indicates a significant improvement in sound attenuation of up to $4 \mathrm{dBA}$. The values are similar and even superior to results reported by other authors regarding the effectiveness of absorptive treatments applied to parallel barriers, and furthermore, the proposed barriers offer an aesthetic element for environmental integration.
\end{abstract}

Keywords: vertical greenery systems, sound absorption, noise barriers, predicted noise attenuation 


\section{Introduction}

Urban planning considers various aspects related to demographics, safety, mobility, and economic viability, among other factors; however, noise control is not always included, at least in the early stages. In such cases, solutions to noise problems must be developed when they appear, typically by reducing the level of noise or obstructing its propagation. Road traffic is one of the most common sources of noise pollution affecting residents' quality of life, especially in an urban context (Kang, 2006; Den Boer and Schroten, 2007). Areas near roads with heavy traffic can be protected by erecting noise barriers. Extensive research on noise barriers with different characteristics has been performed over the past four decades, including geometrical analyses, theoretical modelling and full-scale testing (Ekici and Bougdah, 2003; Kotzen and English, 2009). Urban development and growth can necessitate the protection of areas to both sides of a heavily trafficked roadway by means of the construction of a pair of acoustic barriers, but in such a case, the multiple reflections occurring between the barriers may result in a significant degradation in screening performance compared with that of a single barrier (Watts, 1996a; Cianfrini et al., 2007; Daltrop and Hodgson, 2012). Watts (1996) performed full-scale tests of several different parallel-barrier configurations and found, for example, that the screening performance of a single $2 \mathrm{~m}$ high barrier on one side of the road was reduced by 4 $\mathrm{dB}(\mathrm{A})$ when a reflective barrier of a similar height was erected on the other side of the road. To improve the performance of noise barriers, various approaches or combinations of approaches can be adopted. Some strategies for mitigating the problems of parallel barriers involve inclining them vertically or designing shapes with which to modify their diffracting edges (Watts, 1996b; Menounou and Busch-Vishniac, 2000; Ishizuka and Fujiwara, 2004). Another method involves the application of sound-absorbing materials to the traffic-facing side of each barrier (Watts, 1996a; Watts and Godfrey, 1999; Palma and Samagaio 2006; Cianfrini, et al., 2007; Daltrop and Hodgson, 2012). As an example, measurements comparing the performance of sound-absorptive, reflective, and T-profile noise barriers (May and Osman, 1980) have shown that a T-profile barrier produces a reduction in the level of noise that is approximately 1- 
$1.5 \mathrm{~dB}(\mathrm{~A})$ greater than that of the same barrier without the cap, whereas no significant difference was observed between the noise reductions achieved in the absorptive and reflective configurations. Nevertheless, absorptive materials can eliminate, or at least reduce, reflection effects and therefore counteract the degradation in the performance of a single barrier caused by the presence of a second parallel barrier (Watts, 1996a). In a scale model (Daltrop and Hodgson, 2012), the effects of barrier absorption on the source sides of barriers of various heights were tested, including the cases of reflective and absorptive parallel barriers. It was shown that the effect of adding absorptive material to the source sides of parallel barriers was equivalent to that of increasing the barrier height by $0.33 \mathrm{~m}$.

A reduction in road traffic noise can be achieved through the optimal use of vegetation (Defrance et al., 2015). The absorptive and scattering properties of greenery make it a potentially suitable alternative to classical barriers, in addition to presenting an aesthetic effect (Ekici and Bougdah, 2003). Moreover, interaction with green spaces has a beneficial impact on noise perception (Dzhambov and Dimitrova, 2015). Although vegetation barriers are able to reduce noise, they must be at least 1.5 metres thick to achieve attenuations higher than $5 \mathrm{~dB}(\mathrm{~A})$ (Kalansuriya et al., 2009). However, a combination of vegetation and a solid barrier may be an interesting and effective solution (Dunnett and Kingsbury, 2004; Wong et al., 2010; Horoshenkov et al., 2013; Daltrop et al., 2012, Hodgson et al., 2013). A recent review paper by Van Renterghem at al. (2015) shows that there are many methods of reducing traffic noise by means of natural barriers between the road and the noise-affected region. Increasing the absorption at the surfaces of parallel road-noise-shielding walls by means of planted substrates is a useful technique. The reflective, deflective and absorptive properties of the plants and the attenuation effects produced by the air between the vegetation and the wall can increase the acoustic isolation provided by such a barrier. Furthermore, the plants and the wall represent opposite spectral solutions for noise transmission, blocking the sound in the lower and higher frequency ranges, respectively (Dunnett and Kingsbury, 2004). Wong et al. (2010) analysed 8 vertical greenery systems. Their results showed stronger attenuation at low to moderate 
frequencies due to the absorbing effect of the substrate, whereas weaker attenuation was observed in the high-frequency spectrum. They also observed an increase in the sound absorption coefficients with greater greenery coverage. Horoshenkov et al. (2013) measured the acoustic absorption coefficients of five types of low-growing plants in the presence and absence of soil. They found that the absorption coefficients of plants are predominantly determined by the area density and the angular orientation of the leaves. The presence of porous soil in which a plant is growing can also affect the ability of the plant to absorb sound because of the interaction between the sound waves propagated through the plant to the top layer of the soil and the sound waves reflected from the porous soil. Yang et al. (2013) considered various factors such as soil depth, soil moisture and level of vegetation. Measurements without vegetation revealed high absorption coefficients, with a significant decrease as the soil moisture increased. The absorption coefficients measured in the presence of plants showed a tendency to increase with increasing vegetation coverage and density.

In previous work, a greenery wall prototype for use in building façades was designed and constructed (Urretarazu and Bures, 2012). The system is composed of independent modular units and a support mechanism. Each modular unit is a closed box filled with a substrate, in which plant species are cultivated. To analyse the possible application of this modular greenery wall for noise reduction, two different standardized laboratory tests were conducted (Azkorra et al., 2015). The main results were a weighted sound reduction index (Rw) of $15 \mathrm{~dB}$ and a weighted sound absorption coefficient $(\alpha)$ of 0.40 . The goal of the present paper is to analyse the performance of this modular-based greenery system when applied in the design of noise barriers to increase their noise absorption capabilities and their integration with their surroundings. First, in situ measurements of noise reflection at a moderate scale were performed to estimate the sound absorption coefficients. Then, the obtained values were input into software for noise prediction to evaluate the potential improvement in sound attenuation that can be achieved by such greenery barriers. 
In Europe, in situ measurements of the sound reflection properties of noise barriers are currently performed in accordance with CEN standards (2003). The specified method involves the averaging of measurements taken at specific angles of incidence. Rotation can be performed in the vertical or horizontal plane. The results are computed in one-third-octave frequency bands and describe the extent to which the device under test reflects a sound wave back towards its source. Recently, in the framework of the European QUIESST project, this method was thoroughly revised (Guidorzi and Garai, 2013; Garai et al., 2014). The method requires a minimum barrier width and height of $4 \mathrm{~m}$ to avoid the effect of ground reflections (if vertical rotation is chosen) or post reflections (if horizontal rotation is chosen). Because of these geometrical constraints, in most cases, the CEN/TS 1793-5 standard (CEN, 2003) cannot be applied. Moreover, to obtain the reflection index for each band, specular reflection properties are assumed. Some authors (Tronchin, 2013) have noted that for this reason, the results obtained using this method may not be adequate for rough surfaces, as this assumption is incorrect for the scattering surfaces. Furthermore, for the in situ measurement of sound absorption coefficients, the ISO 13472-1 standard is recommended for road surfaces. Several authors have applied this technique to various surfaces (Londhe, 2009; Guidorzi, 2008). In the study reported in this paper, a procedure based on this method, although with some differences, was used to compare the reflection characteristics of barriers with and without the proposed modular greenery system.

The noise reduction provided by barriers is often evaluated through numerical simulations. Numerous software packages are available for assisting in the prediction of traffic noise levels and the evaluation of noise barrier performance, such as CadnaA, SoundPlan, LimaA, Immi or MicroBruit (Boczkowski, 2013; Guarnaccia et al., 2014a, 2014b; Carr et al., 2012). In this study, CadnaA (Computer Aided Noise Abatement) was used. The general approach of CadnaA is based on the inverse ray-tracing technique (Guarnaccia et al., 2014a). This software enables the analysis of the noise generated by various sources, such as roads and railways, based on international standard technical parameters. Either localized receivers or extended areas can be 
considered; in the latter case, the analysis is performed for individuals divisions of a grid of the desired size.

\section{Materials and methods}

\subsection{Description of the prototype greenery wall}

The barrier was constructed from concrete blocks and coated with mortar. The dimensions of the prototype barrier are $2.62 \mathrm{~m}$ in width, $2.42 \mathrm{~m}$ in height and $0.20 \mathrm{~m}$ in thickness. The wall is shown with and without greenery in Fig. 1.
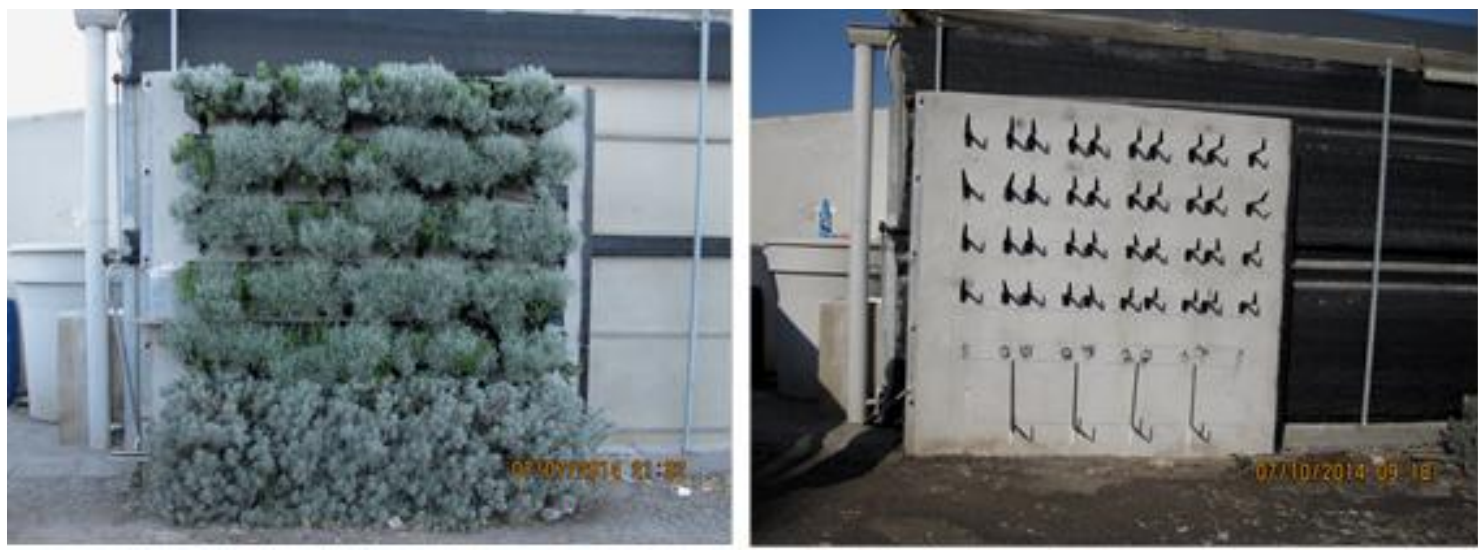

Figure 1. Experimental prototype with and without the modular greenery system

The green wall system is based on recycled polyethylene modules; each is an independent hydroponic crop unit (MCU) (Urrestarazu, 2012; Perez, 2016). The MCUs are resistant to UV radiation and are $600 \mathrm{~mm}$ wide by $200 \mathrm{~mm}$ high by $400 \mathrm{~mm}$ thick. Each MCU is a closed box filled with a recyclable and environmentally friendly substrate: a 50/50 vol/vol mix of compost and coconut fibres of $15 \mathrm{~cm}$ in thickness. A lower layer of recycled plastic (3 $\mathrm{mm}$ in thickness), which is environmentally beneficial by virtue of the recycling of the plastic, also provides resistance and collects rainwater from the upper layer. Each MCU is independent and is held in place by a stainless steel structure. An irrigation system and vegetal canopy, as described by Urrestarazu and Burés (2012) and Azkorra et al. (2015), is used. This green wall is designed to host any small shrub, although native plants that are well adapted to the climate are 
recommended. In this study, the Helichrysum thianschanicum species was used. This species is widely used in gardening in the Mediterranean climate and particularly in plant façades because of its high resistance to drought and high temperatures. Each module contained 24 precultivated plants with an average height of $0.4 \mathrm{~m}$. Acoustic measurements were performed after the irrigation, resulting in a composition of $16.2 \%$ solid materials, $51.8 \%$ water and $23 \%$ air (by volume).

\subsection{In situ measurement procedure}

In situ measurements of sound reflection were performed on the experimental noise barrier both with and without the modular greenery system. Although the small dimensions of the prototype prevented the precise determination of the noise absorption characteristics, a comparative analysis could be performed to quantify the improvement achieved by mounting greenery on the plain concrete wall. A method based on the ISO 13472-1 standard and the European CENT/TS 1793 standard, though with certain simplifications and adaptations to the experimental set-up, was used. The procedure consisted of generating and analysing the evolution of a train of very short acoustic signals (pulses) incident on and reflected by the barrier. A microphone was placed between the omnidirectional source and the barrier (Fig. 2). With the selection of a sufficient short time for analysis, it is possible to assume that this microphone received both the directly incident sound and that reflected by the barrier. In addition, the directly incident sound was also registered by placing a second microphone at the same distance from the noise source but in a location free of reflections coming from nearby walls. The effect of ground reflections is expected to be similar in both recordings. Thus, the subtraction of the two simultaneously recorded signals should yield the signal corresponding to the reflected sound. The characteristics of the devices used are as follows: two M1-17 1/4" microphones and a DO12 omnidirectional source. The barrier and the devices were placed on an earthen floor, which can be regarded as a highly absorptive material. 


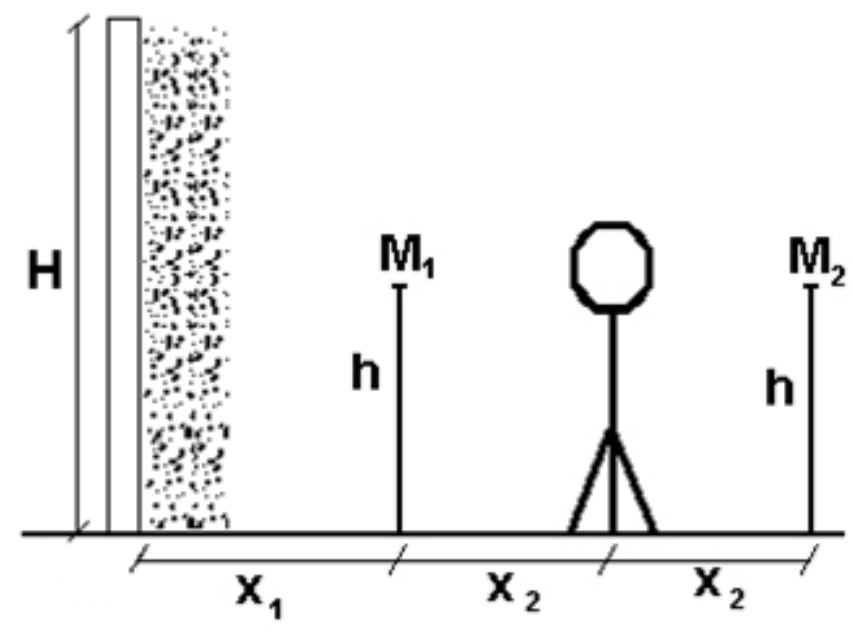

Figure 2. Sketch of the experimental set-up. $\mathrm{H}=2.42 \mathrm{~m}, \mathrm{~h}=0.8,0.9$ and $1 \mathrm{~m}, \mathrm{x} 1=1.4$ and $\mathrm{x} 2=1 \mathrm{~m}$.

As the objective of this study was to quantify the decrease in the reflection component achieved with the use of the developed greenery system, measurements with and without vegetation were performed under otherwise identical conditions. The distance from the noise source to the microphone was $1 \mathrm{~m}$, and that from the microphone to the wall was $1.4 \mathrm{~m}$. It should be noted that this latter distance is greater than the recommended value, but this choice is justified by the large thickness of the greenery elements. For each barrier, the spectral analysis of both the direct and reflected signals, evaluated within a small time window and corrected for the difference in path length, yields the surface reflection factor $R_{P}(f)$ for each octave frequency band:

$$
R_{P}(f)=\frac{1}{K_{r}} \frac{P_{r}(f)}{P_{d}(f)}
$$

where $K_{r}$ is a geometrical factor that depends on the difference between the paths followed by the direct and reflected waves, $P_{r}(f)$ is the spectrum of the sound pressure wave reflected by the barrier (after the application of the subtraction technique), and $P_{d}(f)$ is the spectrum of the direct sound pressure wave. It is worth noting several potential difficulties encountered in both performing the measurements and handling the data. An incorrect determination of geometrical factors and other corrections can lead to strong discrepancies in the reflection factors. A 
significant improvement in the accuracy of the method can be achieved by performing a reference measurement using a totally reflecting surface for the same positions of the loudspeaker and the microphones (Garai et al., 1998). In our case, the wall without greenery is not a totally reflecting surface, but it exhibits a known reflection behaviour and thus can be treated as the reference surface. The relative reflection factor $R_{p, r e l}(f)$ is then evaluated as follows:

$$
R_{p, \text { rel }}(f)=\frac{P_{r, \text { green }}(f)}{P_{r, \text { wall }}(f)}
$$

where $P_{r, \text { green }}(f)$ and $P_{r, \text { wall }}(f)$ are the spectra of the sound pressure waves reflected by the barrier with and without greenery, respectively.

Once the relative reflection factors have been obtained, the relative absorption coefficients can be calculated as follows:

$$
\alpha_{r e l}(f)=1-\left|R_{P, r e l}(f)\right|^{2}
$$

The real absorption coefficients for the greenery wall can then be estimated from the relative coefficients $\alpha_{\text {rel }}$ and the known absorption coefficients of the reference barrier, $\alpha_{\text {wall }}$ :

$$
\begin{aligned}
\alpha(f) & =1-\left|R_{P}(f)\right|^{2}=1-\left|R_{P, \text { wall }}(f)\right|^{2} \cdot\left(\alpha_{\text {rel }}(f)-1\right) \\
& =\alpha_{\text {rel }}(f)+\alpha_{\text {wall }}(f) \cdot\left(1-\alpha_{\text {rel }}(f)\right)
\end{aligned}
$$

In accordance with this procedure, a train of identical pulses was generated, each with a duration of $3 \mathrm{~ms}$ and separated by $1 \mathrm{~s}$. The microphone was always placed at a distance of $1.4 \mathrm{~m}$ from the wall, but three different heights were tested: $\mathrm{h}=100,90$ and $80 \mathrm{~cm}$. As observed from Fig. 1, the greenery system was quite heterogeneous. The heights were selected such that points at $\mathrm{h}=100 \mathrm{~cm}$ were in front of zones with dense vegetation, whereas points at $\mathrm{h}=80$ were in front of zones with sparse vegetation. Differences in the results for the different heights could 
therefore provide information about the dependence of the sound reflection behaviour on the vegetation density.

\subsection{Procedure for the numerical prediction of attenuation levels}

The environmental noise prediction software CadnaA was employed to evaluate the improvement that can be achieved by using greenery barriers instead of barriers with low absorption. Calculations were performed for a 6-lane motorway of $30 \mathrm{~m}$ in width with an average daily traffic (ADT) of 12000 vehicles. The barrier height was set to $\mathrm{H}=3 \mathrm{~m}$, and each was placed at a distance of $\mathrm{W} / 2$ from the centre of the track. Barriers regarded as "reflective barriers" were characterized by a low absorption coefficient (a value of 0.2 was set for all frequencies), whereas "greenery barriers" were assigned the absorption values measured from the modular greenery prototype.

Receivers were placed at distances $d$ from the barrier and at heights $h$ from the ground. At each receiver position, the improvement achieved for the green barrier, $\Delta L_{A}$, was calculated as follows:

$$
\Delta L_{A}=L_{A, \text { ref }}-L_{A, \text { green }}
$$

where $L_{A, \text { ref }}$ and $L_{A, \text { green }}$ are the sound levels, in $\mathrm{dB}(\mathrm{A})$, for pairs of reflective and greenery barriers, respectively.

\section{Results}

\subsection{In situ measurement results}

As described in Section 2.2, two microphones received a train of identical pulses representing both direct and reflected signals at various heights. As an example, Fig. 3 (top panel) shows the temporal evolution of the signals recorded by the microphone located at a height of $h=100 \mathrm{~cm}$ for the barrier with and without greenery. In the case of the greenery-free barrier, reflection of the sound is clearly seen (dashed line), with a delay of approximately $8 \mathrm{~ms}$ from the directly 
incident wave. In the presence of the greenery system, reflection is also observed but, as expected, at a much lower level (solid line). The reflected waves can be obtained by subtracting from these signals $\left(\mathrm{p}_{\mathrm{t}}\right)$ the directed signals $\left(\mathrm{p}_{\mathrm{d}}\right)$ recorded by a microphone at the same distance from the noise source but in a location free of reflections from nearby walls. The reflected signals $\left(p_{r}=p_{t}-p_{d}\right)$ are presented in Fig. 3 (bottom panel). In this representation, the effect of the vegetation in reducing the reflection of the wave can be more clearly observed. The first reflection from the barrier arrives at the second microphone $14 \mathrm{~ms}$ later than the direct signal. Therefore, in light of the observed signals, a time window of $10 \mathrm{~ms}$ (also shown in Fig. 3) appears adequate for further analysis.

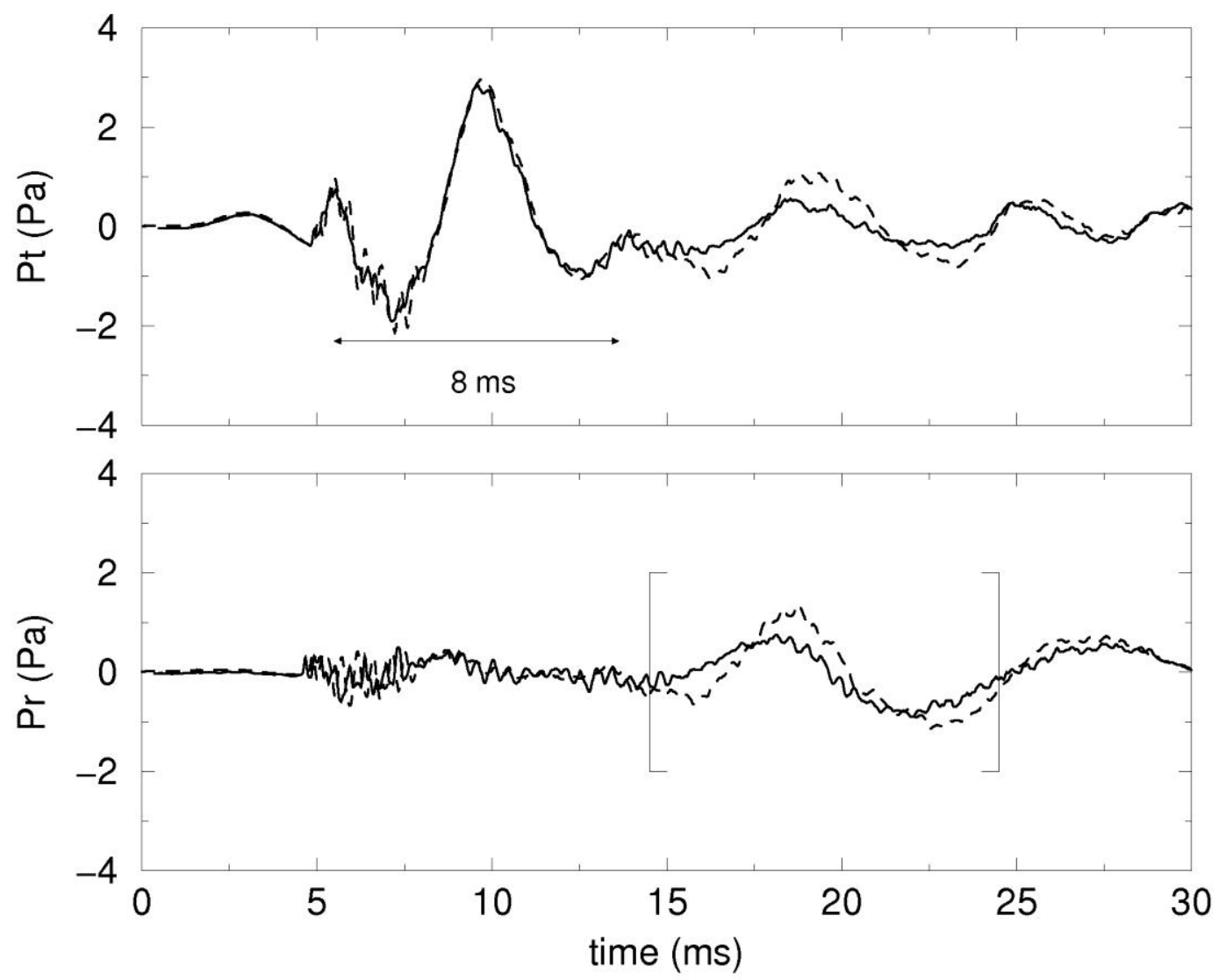

Figure 3. Top panel: Signals recorded by a microphone placed between the sound source and the barrier at a height of $\mathrm{h}=100 \mathrm{~cm}$ from the ground. Bottom panel: The reflected signals obtained by subtracting the direct signals. Solid lines correspond to the greenery-covered barrier, and dashed lines correspond to the reference barrier without greenery. The selected time window is indicated in the bottom panel. 
Fig. 4 presents the sound pressure levels averaged over all analysed pulses that correspond to the reflected waves, in octave bands. The three left panels compare the levels for the barrier with and without greenery for the three microphone heights. In each case, the mean value obtained from 6 recorded pulses is shown. As expected, the levels corresponding to the greenery-free wall are always higher than those measured for the wall with greenery. The difference between the two values depends on the height of the microphone and is highest for the case of $\mathrm{h}=100 \mathrm{~cm}$. This is because, as previously mentioned, the vegetation was distributed quite heterogeneously, and this reception point was immediately in front of a zone of dense vegetation. The right panels of Fig. 4 show, for each height, the relative absorption

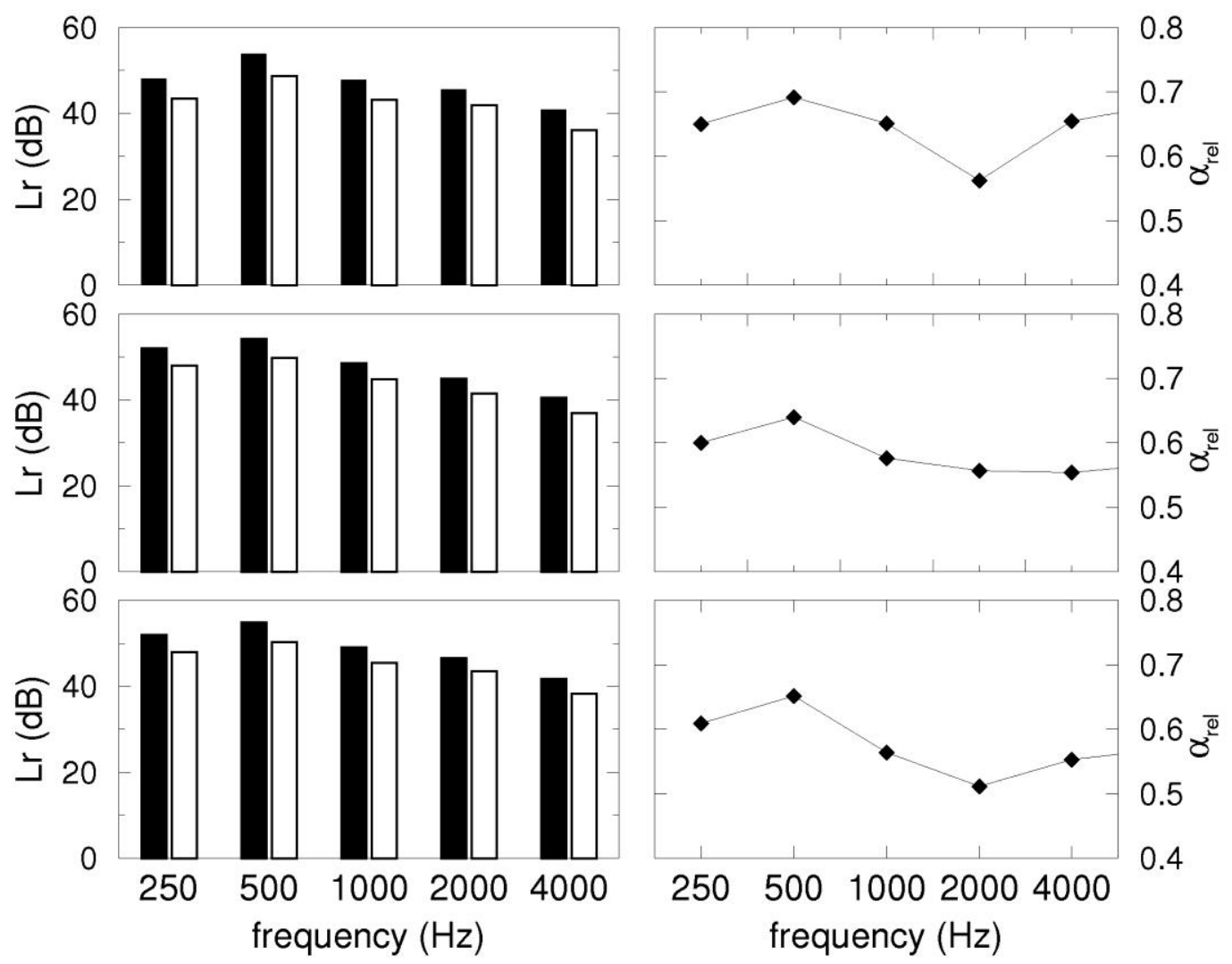

Figure 4. Left panels: The reflected sound levels for the reference barrier without greenery (black) and the greenery-covered barrier (white) at three different microphone positions: $\mathrm{h}=100 \mathrm{~cm}$ (top), $90 \mathrm{~cm}$ (middle) and $80 \mathrm{~cm}$ (bottom). Right panels: The corresponding relative absorption coefficients. 
coefficients calculated using Eqs. (2) and (3). We observe that these relative coefficients are higher than 0.5 for all frequencies and measurement points. The standard deviation is 0.05 . Table 1 shows the relative absorption coefficients, averaged over the three microphone positions, and the real absorption coefficients $\alpha$ of the greenery barrier calculated using Eq. (4).

Table 1. Relative absorption coefficients averaged over the three positions of the microphone. The absorption coefficients $\alpha$ were estimated via Eq. (4) using the reference absorption characteristics for a low-absorption surface (also shown in the table).

\begin{tabular}{cccccc}
\hline & $250 \mathrm{~Hz}$ & $500 \mathrm{~Hz}$ & $1000 \mathrm{~Hz}$ & $2000 \mathrm{~Hz}$ & $4000 \mathrm{~Hz}$ \\
\hline$\alpha$ rel & 0.65 & 0.68 & 0.63 & 0.56 & 0.64 \\
$\alpha$ wall & 0.20 & 0.20 & 0.20 & 0.20 & 0.20 \\
$\alpha$ & 0.70 & 0.73 & 0.67 & 0.63 & 0.66 \\
\hline
\end{tabular}

The results obtained for the absorption coefficients in the range of moderate and high frequencies indicate values of approximately 0.65 . At lower frequencies, the absorption is higher, but it should be noted that because of the small value of the temporal window used in this analysis, the absorption coefficients at such low frequencies may be subject to error.

\subsection{Predicted attenuation improvement provided by greenery-covered noise barriers}

As explained in the introduction, when a pair of noise barriers is erected to protect areas located near roadways, the multiple reflections occurring between the barriers may result in significant degradation of the attenuation performance compared with that of a single barrier. It is expected that this problem should be minimized by the use of greenery barriers, and the objective of this section is to quantify this improvement. The environmental noise prediction software CadnaA was employed for this purpose, following the method explained in Section 2.3. First, the separation between the barriers was set to $\mathrm{W}=46 \mathrm{~m}$. Several sets of receivers, 
located at different distances $\mathrm{d}$ from the barrier and at different heights $\mathrm{h}$ from the ground, were considered. Fig. 5 shows the predicted sound maps, at three different heights, corresponding to configurations with a single barrier, a pair of parallel reflective barriers and a pair of parallel greenery barriers.
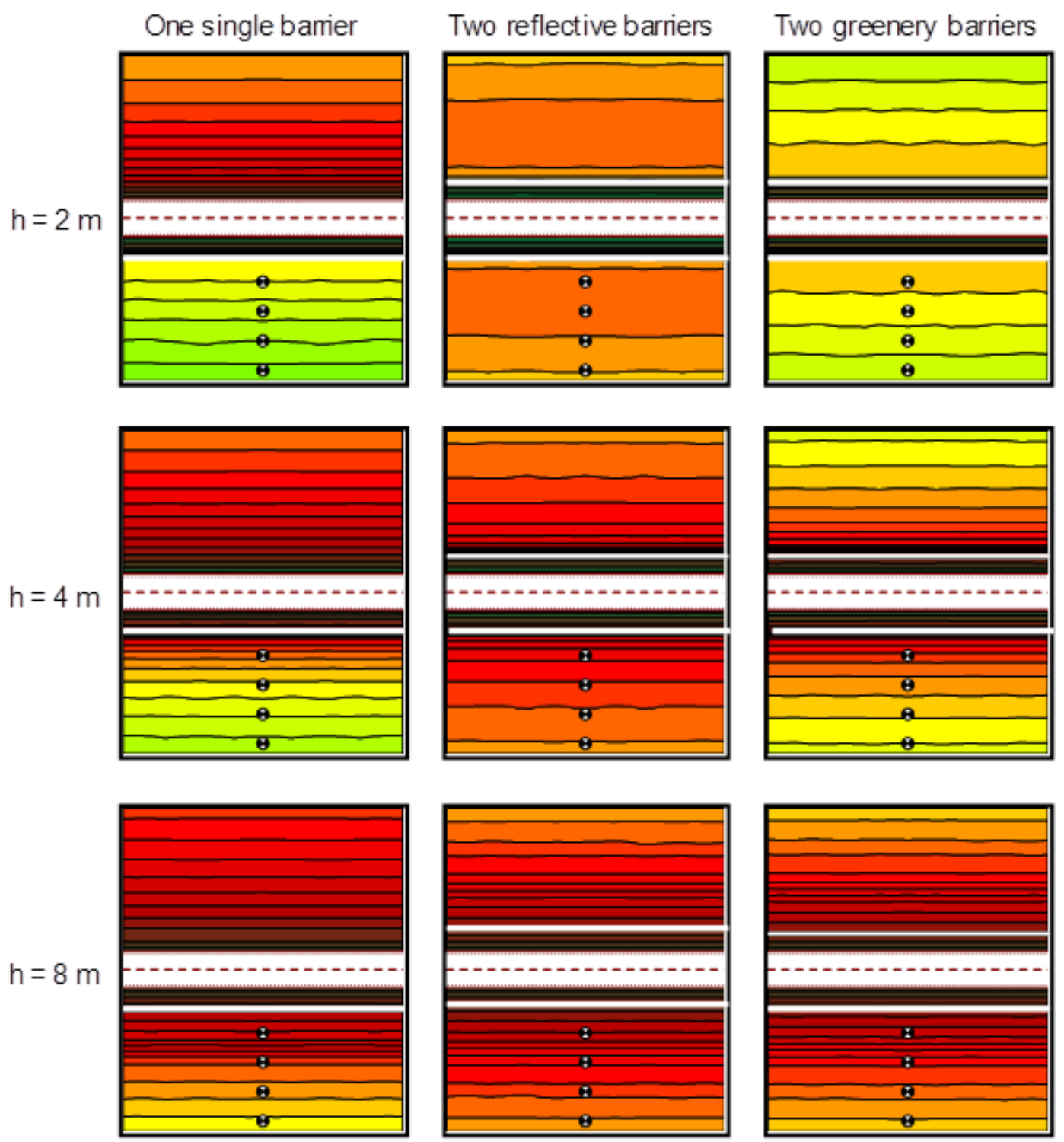

Figure 5. Predicted sound maps at three heights with respect to ground level, $h=2,4$ and $8 \mathrm{~m}$, and three barrier configurations (shown in white). Left panels: One reflective barrier placed $23 \mathrm{~m}$ from the centre of the track. Central panels: Two reflective barriers, each $23 \mathrm{~m}$ from the centre of the track (W=46 m). Right panels: Same as the central panels but with greenery barriers. Colours from red to green indicate a decrease in noise level.

The single-barrier case presented in the figure (left panel) corresponds to a reflective barrier, but in fact, the attenuation experienced behind a single barrier does not depend significantly on the 
absorption coefficient. The colours in the figure illustrate how sound is attenuated behind the barrier; sound levels lower than those in front of the barrier are indicated, especially at a height of $\mathrm{h}=2 \mathrm{~m}$. When a second reflective barrier is added on the other side of the road (central panels), the occurrence of multiple reflections increases the noise level from the road, causing a clear reduction in the attenuation behind the first barrier (the green colours shift to orange tones). However, when a pair of greenery barriers instead of reflective barriers is erected (right panels), the attenuation obtained behind the single barrier appears to be recovered.

To better understand the behaviour shown in Fig. 5, the sound levels at a height of $\mathrm{h}=4 \mathrm{~m}$ are plotted as a function of the distance from the barrier in Fig. 6. A comparison of the results for the three analysed configurations shows that the use of greenery barriers considerably mitigates the reflection effect, although it does not eliminate it completely. The improvement, calculated using Eq. (5), is shown in the inset of Fig. 6. It increases with increasing distance and is equal to approximately $2.3 \mathrm{dBA}$ at a distance of $\mathrm{d}=40 \mathrm{~m}$. For this case $(\mathrm{h}=4 \mathrm{~m}$ and $\mathrm{d}=40 \mathrm{~m})$, Fig. 7 compares the sound levels in the various frequency bands.

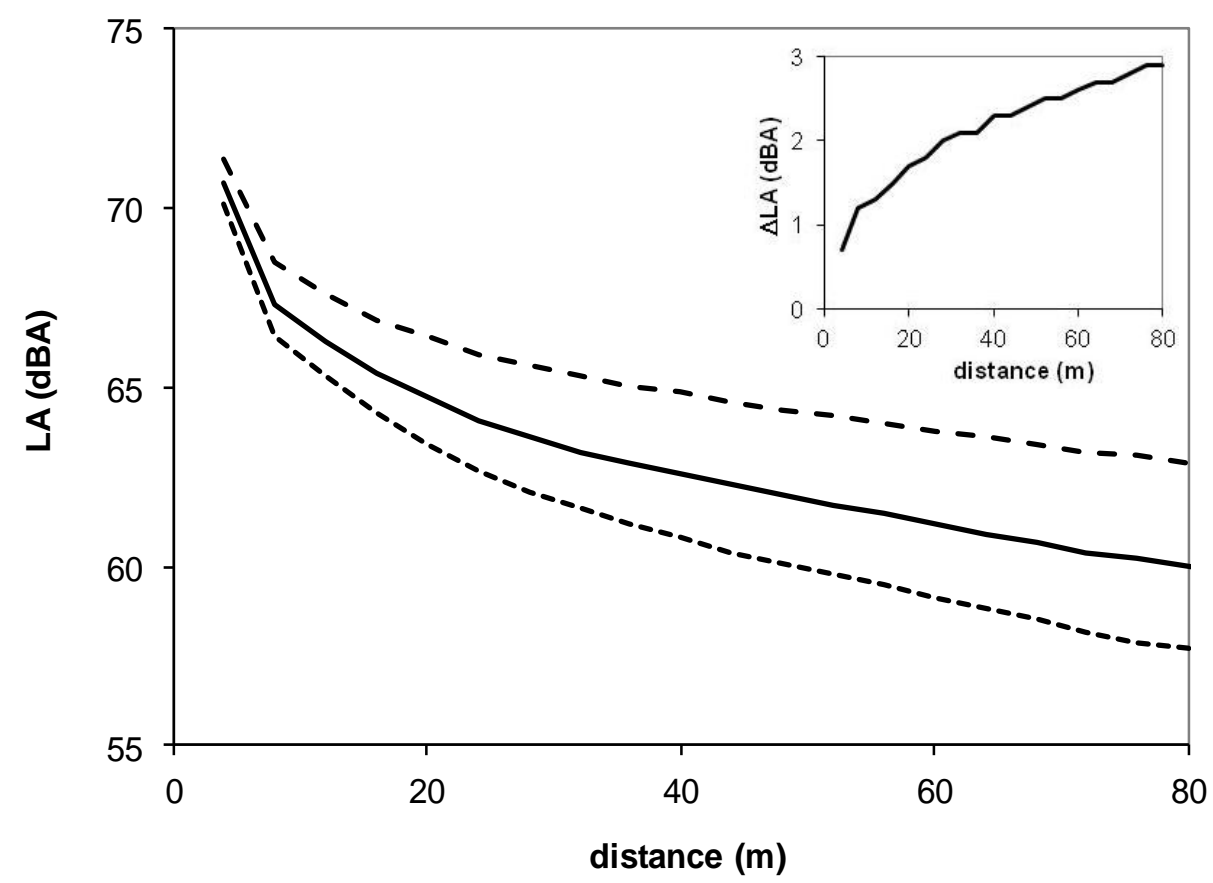


Figure 6. Predicted sound levels as functions of the distance from the barrier for a single reflective barrier (dotted line), a pair of reflective barriers (dashed line) and a pair of greenery barriers (solid line). The receiver height is $\mathrm{h}=4 \mathrm{~m}$. Inset: The improvement $\Delta \mathrm{L}_{\mathrm{A}}$ (Eq. (5)) achieved by using greenery barriers instead of a pair of reflective barriers.

In Fig. 8, the improvements achieved by using greenery barriers are presented, as functions of the receiver height $h$, for distances of $d=20,40,60$ and $80 \mathrm{~m}$ (these positions are marked in the sound maps presented in Fig. 5). As expected, the difference between using reflective or absorbing barriers is less important at greater heights. Above $8 \mathrm{~m}$, the improvement is only approximately $1 \mathrm{~dB}(\mathrm{~A})$ or less. At short distances $(\mathrm{d}=20 \mathrm{~m})$, the improvement reaches a maximum at a height approximately equal to the barrier height $(3 \mathrm{~m})$. At longer distances, this maximum occurs at a lower height.

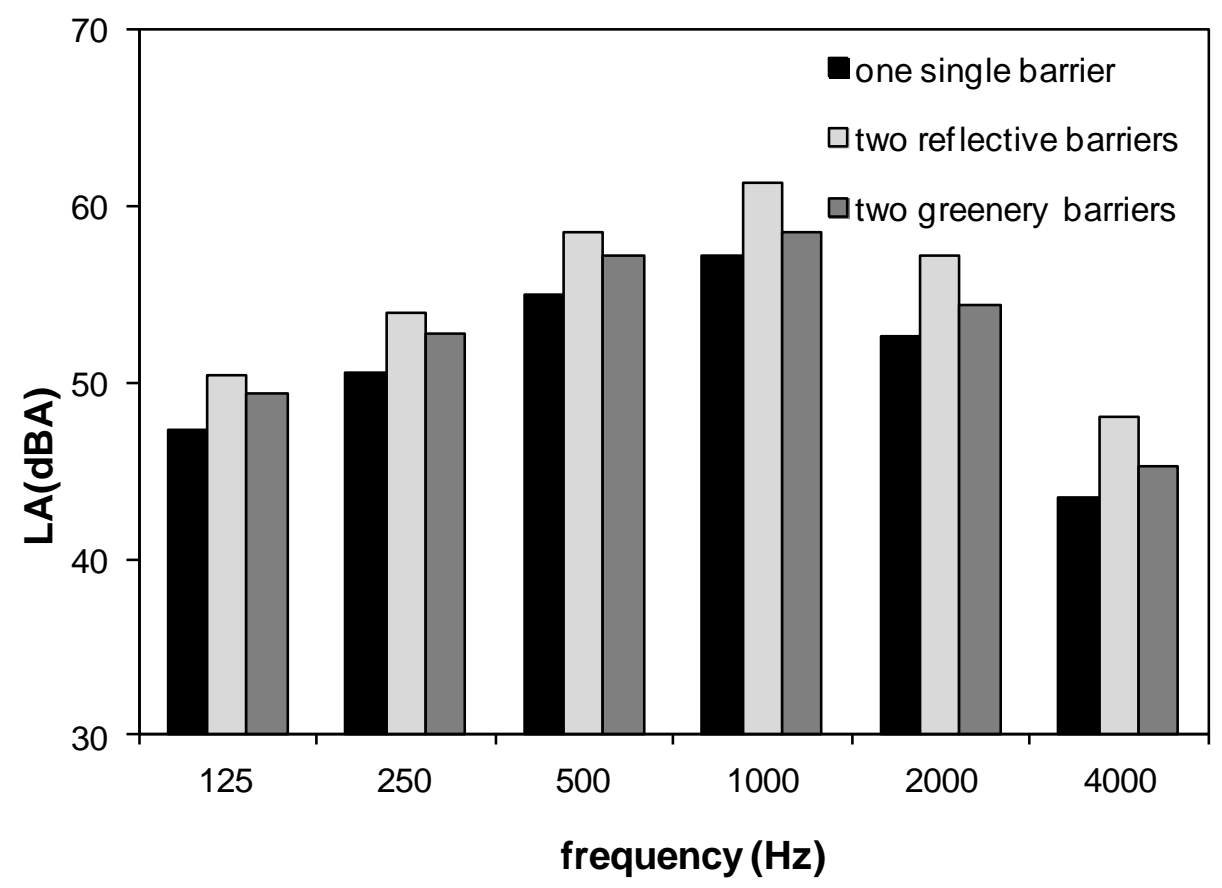

Fig. 7. Predicted sound-level distributions, in octave frequency bands, for a receiver at a height of $h=4 \mathrm{~m}$ and a distance from the barrier of $\mathrm{d}=40 \mathrm{~m}$. The three different barrier configurations are compared. 


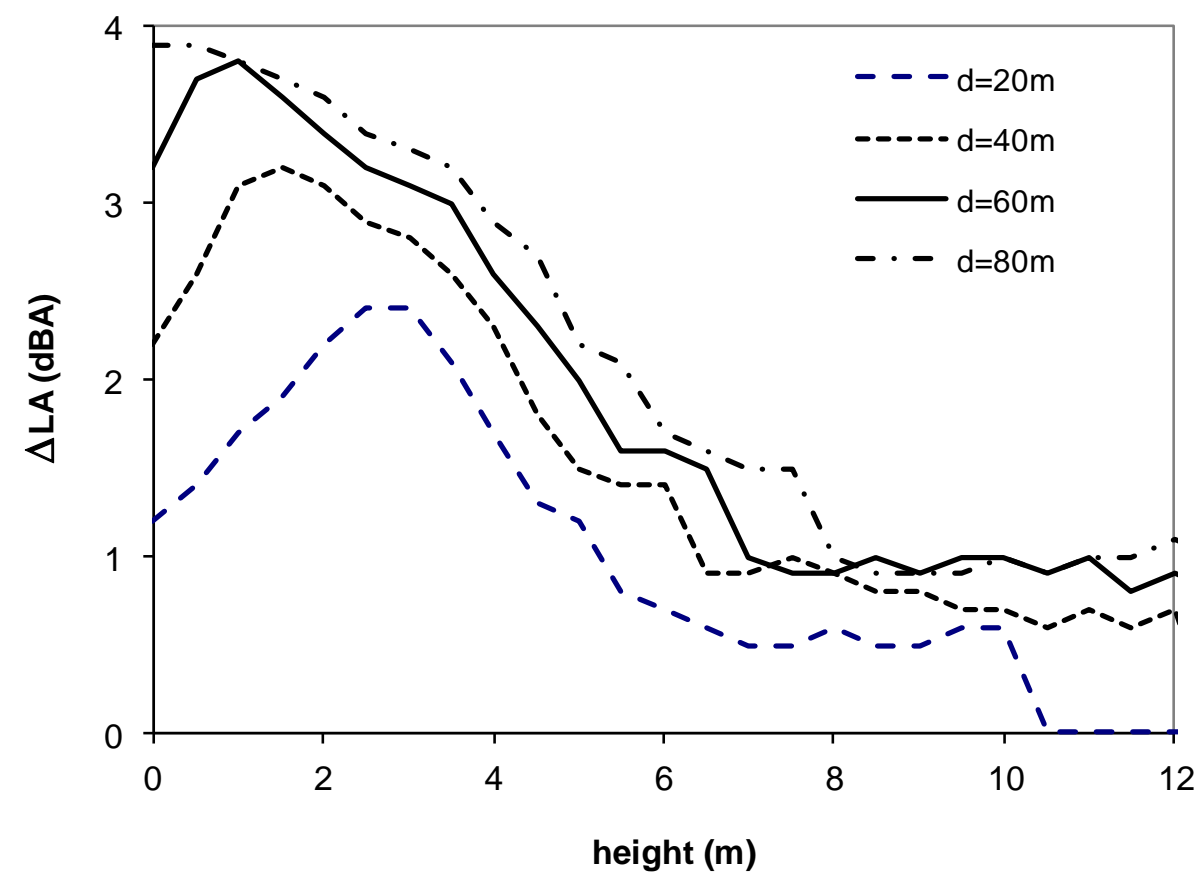

Figure 8. Predicted improvements achieved using a pair of greenery barriers, as functions of height, for distances of $\mathrm{d}=20,40,60$ and $80 \mathrm{~m}$.

Finally, we study the dependence on the separation between the barriers, W. Three separations are considered: $\mathrm{W}=38,46$ and $54 \mathrm{~m}$. Because the barrier height is fixed at $3 \mathrm{~m}$, these separations correspond to barrier height ratios, $\mathrm{W} / \mathrm{H}$, of approximately 12,15 and 18 , respectively. The results are shown in Figs. 9 and 10. In Fig. 9, the sound levels for reflective and greenery barriers obtained at a height of $h=4 \mathrm{~m}$ for each of the three separations are plotted as functions of the distance from the barrier. As expected, when the separation is smaller, the effect of reflections is more important and, therefore, the improvement offered by the greenery barriers is higher. Fig. 10 compares the improvements, as functions of height, for a receiver located $40 \mathrm{~m}$ from the barrier. Is clearly seen that a lower separation yields greater improvement. 


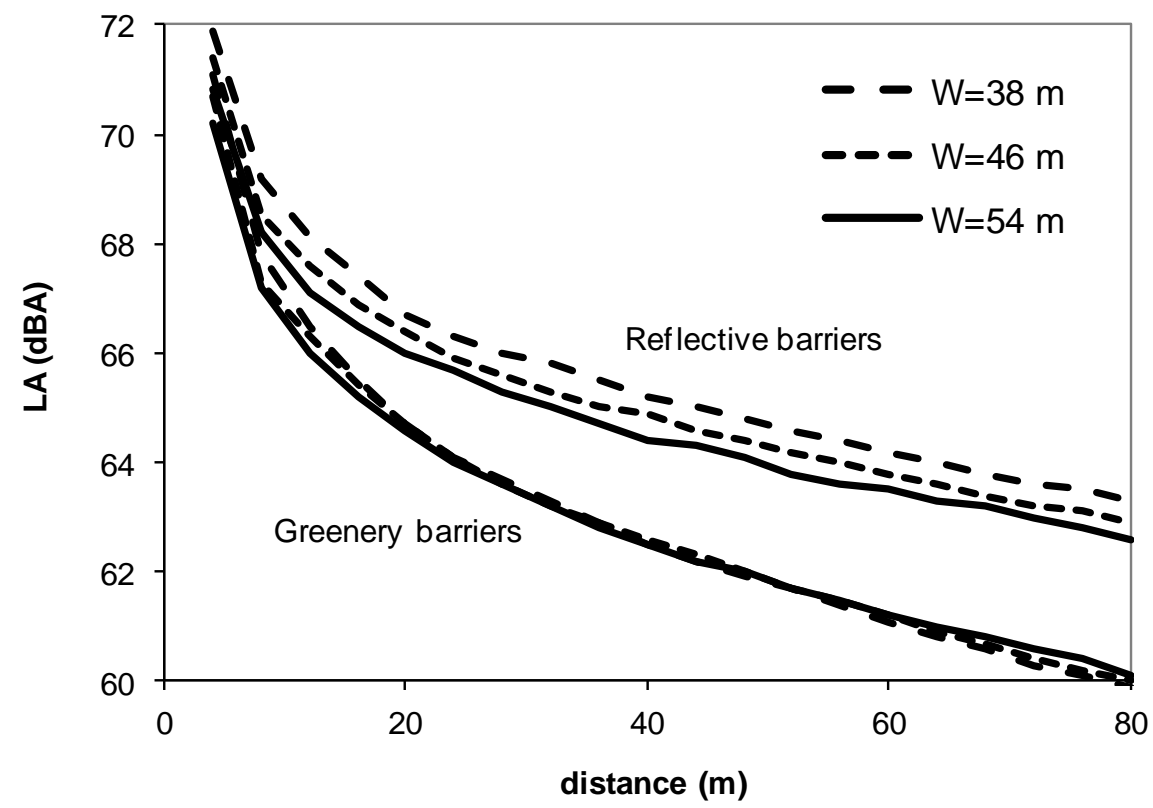

Figure 9. Predicted sound levels for pairs of reflective and greenery barriers with separations of $\mathrm{W}=38,46$ and $54 \mathrm{~m}$ as functions of the distance from the barrier. The receiver height is $\mathrm{h}=4 \mathrm{~m}$.

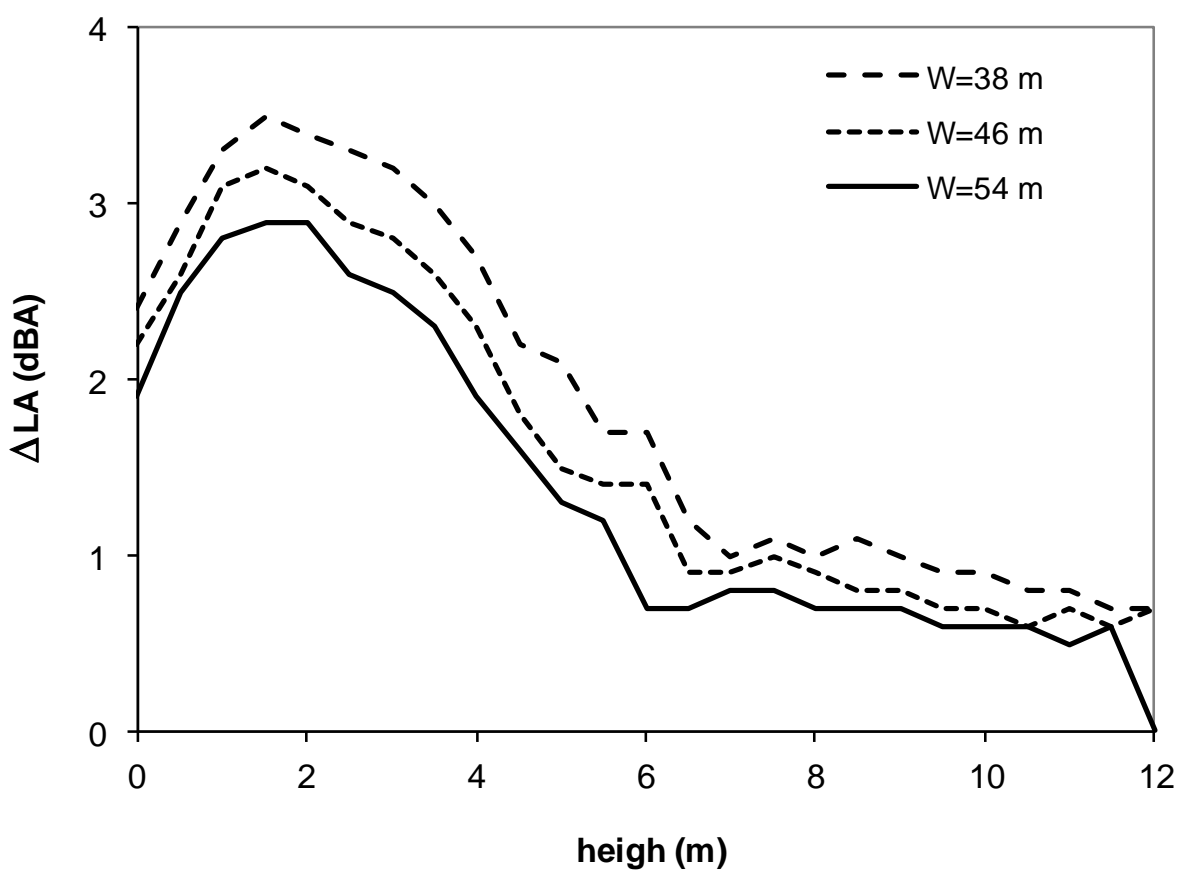

Figure 10. Predicted improvements for pairs of greenery barriers with separations of W=38, 46 and $54 \mathrm{~m}$ as functions of height. The receiver is placed at a distance of $\mathrm{d}=40 \mathrm{~m}$. 


\section{Discussion and conclusions}

A modular greenery wall was acoustically characterized to evaluate its potential performance as a roadway noise barrier. First, in situ measurements of the sound reflection and absorption coefficients were performed using an experimental prototype. The results for the greenerycovered wall and a reference wall without greenery were compared, revealing a clear improvement in the presence of greenery. The in situ measured absorption coefficients have an average value of approximately 0.7 . In general, the values for all frequency bands are higher than those previously obtained in laboratory measurements (Azcorra et al., 2015), which can be attributed to the fact that in these previous measurements, the vegetation in the greenery system was not as highly developed as that in the prototype barrier used in this study. In fact, these findings reinforce our belief that in situ measurements of this kind are a valuable tool for the realistic evaluation of greenery walls, which exhibit significant changes throughout the year that cannot be conveniently evaluated in the laboratory.

The obtained noise absorption results are reasonable considering the kinds of substrate and vegetation used. The values are higher than those obtained by other authors with high-density substratum soil but lower than those obtained for low-density substratum soil (Horosenkov et al., 2013). The results are also in qualitative agreement with the values obtained by Wong et al. (2010) for greenery with a high coverage density. In addition, the values are similar to or even higher than those obtained by other authors for porous concretes developed for highway noise barriers that incorporate recycled waste materials, such as coal bottom ash (Arenas et al., 2013) and waste concrete (Park et al., 2005), as coarsening aggregates. 
Numerical predictions obtained using the CadnaA software regarding the effectiveness of the proposed greenery system indicate that this system enables an improvement in noise-blocking performance when used in a pair of parallel barriers. The multiple reflections between the barriers are effectively minimized by the absorption provided by the greenery. The achieved improvement depends on the receiver position and the distance between the barriers, but a value of $4 \mathrm{~dB}(\mathrm{~A})$ was obtained as a maximum for realistic situations. A comparison with the results obtained by other authors for absorptive parallel barriers is difficult because of the dependence on the specific characteristics of the barriers (dimensions, barrier separation), the traffic noise considered in each study and the receiver positions. However, the obtained improvement appears similar to that reported by some authors (Watts, 1996b) and higher than that reported by others (Daltrop and Hodgson, 2012).

The results obtained in this work are somewhat limited because they correspond to a particular level of vegetation growth in a system that varies throughout the year. However, when the experimental evaluation was performed, the vegetation was in an intermediate stage, and thus, the noise absorption values reported here can be regarded as average values. In any case, it is worth noting that unlike laboratory experiments, the proposed in situ protocol can be applied to collect measurements in different seasons and thus to evaluate the temporal evolution of the noise absorbance caused by the growth of the vegetation in green barriers. Additionally, the effects of different substrates could be analysed in future works. Another potential extension of this work, relevant for urban planning, would be to consider numerical predictions for a more realistic urban scenario. As shown in this paper, numerical simulations allow a greater understanding of the soundscapes in environments with various noise sources and could be a useful tool for facilitating the consideration of noise problems in urban planning projects to predict the future effects of sources of disturbance. 
In conclusion, the proposed greenery barrier can be an alternative to other types of conventional barriers because, in addition to its aesthetic value, its high sound absorption provides an effective reduction in noise levels. The visualization of the results obtained through simulation clarified the distributions of the noise sound levels in all areas surrounding a road or highway for barriers with different configurations and characteristics. Furthermore, the applied in situ method is suitable for investigating the effects of changes in vegetation and enables the study of barriers with reduced dimensions.

\section{Acknowledgements}

The authors would like to thank the FEDER of the European Union for financial support through the project "G-GI3000/IDIX SILENTVEG: Barreras vegetales autónomas y sostenibles para la mitigación acústica y compensación del $\mathrm{CO} 2$ en vías de transporte, con seguimiento telemático" of the "Programa Operativo FEDER de Andalucía 2007-2013”. 


\section{References}

Arenas, C., Vilchez, L. F., Leiva, C., , H, Rodríguez-Galán, M. Use of co-combustion bottom ash to design an acoustic absorbing material for highway noise barriers. Waste Management 33 (2013), pp. 2316-2321.

Azkorra, Z., Pérez, G., Coma, J., Cabeza, L. F., Bures, S., Álvaro, J. E., Urrestarazu, M.,. Evaluation of green walls as a passive acoustic insulation system for buildings. Applied Acoustics 89 (2015), pp. 46-56.

Boczkowski, A. Some observations on the design of noise barriers. Management Systems in Productions Engineering. Scientific and technical quarterly, 2(10) (2013) pp. 32-36.

Carr, K., Penton, S., Li, M. Evaluation of road transportation noise modelling algorithms and software packages. Canadian Acoustics, 39(3) (2011), pp. 84-85.

CEN/TS 1793-5:2003. 2003. Road traffic noise reducing devices - Test method for determining the acoustic performance -Part 5: Intrinsic characteristics - In situ values of airborne sound reflection and airborne sound insulation.

Cianfrini, C., Corcione, M., Fontana, L. Experimental verification of the acoustic performance of diffusive road side noise barriers. Applied acoustics, 68(11) (2007), pp. 1357-1372.

Daltrop, S. and Hodgson, M. Scale-model investigation of the effects of surface absorption and nearby foliage on noise-barier performance. Canadian Acoustics, 40(4) (2012), pp. 41-48.

Daltrop, S., Hodgson, M. Wakefield, C., Field investigation of the effects of vegetation on the performance of roadside noise barriers. Noise Cont. Eng. J. 60(2) (2012), pp. 202-208.

Defrance, Jérôme, et al. Innovative barriers in Environmental methods for transport noise reduction, Nilsson, M., Bengtsson, J. and Klæboe, R. (Eds.), CRC Press (2014), pp. 1947.

Den Boer, L. C., and Schroten, A. Traffic noise reduction in Europe. CE Delft 14 (2007), pp. 2057-2068.

Dunnett, N., Kingsbury, N. Planting green roofs and living walls (Vol. 254). Portland, OR: Timber Press (2004). 
Dzhambov, Angel. M., and Dimitrova, Donka D. Green spaces and environmental noise perception. Urban Forestry and Urban Greening, 14(4) (2015) pp. 1000-1008.

Ekici, I., Bougdah, H., 2003. A review of research on environmental noise barriers. Building Acoustics, 10(4) (2003), pp. 289-323.

Garai, M., Berengier, M., Guidorzi, P., L’Hermite, P., 1998. Procedure for measuring the sound absorption of road surfaces in-situ. In Proceedings of Euronoise, 98 (1998), pp. 819-824.

Garai, M., Schoen, E., Behler, G., Bragado, B., Chudalla, M., Conter, M., Guidorzi, P. Repeatability and Reproducibility of In Situ Measurements of Sound Reflection and Airborne Sound Insulation Index of Noise Barriers. In Acta Acustica united with Acustica, 100(6) (2014), pp. 1186-1201.

Guarnaccia, C., Quartieri, J., Mastorakis, N. E. Comparison of Acoustic Barriers Noise Reductions Evaluated by Different Calculation Methods. In Latest Trends on Systems", Proc. of the 18th Int. Conf. on Circuits, Systems, Communications and Computers (2014a), pp. 443-449.

Guarnaccia, C., Quartieri, J., Mastorakis, N. E. Analysis of Methods to Evaluate the Noise Reduction due to Acoustic Barriers Installation, International Journal of Mechanics 8 (2014b), pp. 258-267.

Guidorzi P., Garai M. Signal analysis in the sound absorption measurement procedure: The importance of time subtraction and reference surface corrections. Proceedings of Acoustics`08, Paris, France, ISBN 978-2-9521105-4-9 (2008), pp.1755-1760.

Guidorzi P, Garai M. Advancements in Sound Reflection and Airborne Sound Insulation Measurement on Noise Barriers. Open Journal of Acoustics, 3 (2013), pp. 25-38

Horoshenkov, K. V., Khan, A., \& Benkreira, H. Acoustic properties of low growing plants. The Journal of the Acoustical Society of America, 133(5) (2013), pp.2554-2565.

Hodgson, M., Daltrop, S., Peterson, R., Benedict, P. Compliance and vegetated-barrier acoustical testing in a purpose-built sound-transmission suite, Noise Cont. Eng. J. 61(5) (2013) pp. 481-486. 
Ishizuka, T., Fujiwara, K. Performance of noise barriers with various edge shapes and acoustical conditions. Applied Acoustics, 65(2) (2004), pp. 125-141.

ISO 13472-1. 2002. Measurement of sound absorption properties of road in situ. Extended surface method.

Kalansuriya, C. M., Pannila, A. S., Sonnadara, D.U.J. Effect of roadside vegetation on the reduction of traffic noise levels. Proceedings of the Technical Sessions. Institute of Physics-Sri Lanka, 25 (2009), pp. 1-6.

Kang, J. Urban sound environment. CRC Press, 2006.

Kotzen, Benz, and English, Colin. Environmental noise barriers: a guide to their acoustic and visual design. CRC Press, (2009).

Londhe, N., Rao, M. D., Blough, J. R., 2009. Application of the ISO 13472-1 in situ technique for measuring the acoustic absorption coefficient of grass and artificial turf surfaces, Applied Acoustics 70 (2009), pp. 129-141

May, D. N., and Osman M.M. 1980. The performance of sound absorptive, reflective, and Tprofile noise barriers in Toronto. Journal of Sound and Vibration 71.1 (1980), pp. 65-71.

Menounou, P., Busch-Vishniac, I. J., 2000. Jagged edge noise barriers. Building Acoustics 7(3) (2000), pp. 179-200.

Palma, M. J. C., Samagaio, A., 2006. Acoustic performance of a noise barrier coated with an absorptive material. Noise control engineering journal 54(4) (2006), pp. 245-250.

Park, S.B., Seo, D.S., Lee, J. Studies on the sound absorption characteristics of porous concrete based on the content of recycled aggregate and target void ratio. Cem. Concr. Res., 35 (2005), pp 1846-1854

Pérez G. Coma J., Barreneche C., de Gracia A., Urrestarazu M., Burés S., Cabeza L.F. Acoustic insulation capacity of Vertical Greenery Systems for buildings. Applied Acoustics 110 (2016), pp. 218-226.

Tronchin L. On the acoustic efficiency of road barriers: The Reflection Index, International Journal of Mechanics, Issue 3, Vol. 7(2013), pp 318-326. 
Urrestarazu M., Burés S. Sustainable green walls in architecture. J Food Agric Environ 10 (2012), pp. 792-4.

Van Renterghem, T., Forssén, J., Attenborough, K., Jean, P., Defrance, J., Hornikx, M., and Kang, J., 2015. Using natural means to reduce surface transport noise during propagation outdoors. Applied Acoustics, 92 (2015), pp. 86-101.

Watts, G. R., Acoustic performance of a multiple edge noise barrier profile at motorway sites. Applied Acoustics 47(1) (1996b), pp. 47-66.

Watts, G. R., Godfrey, N. S. Effects on roadside noise levels of sound absorptive materials in noise barriers. Applied Acoustics 58(4) (1999), pp. 385-402.

Watts, G.R.,. Acoustic performance of parallel traffic noise barriers. Applied Acoustics 47(2) (1996a), pp. 95-119.

Wong, N. H., Kwang Tan, A. Y., Tan, P. Y., Chiang, K., Wong, N. C. Acoustics evaluation of vertical greenery systems for building walls. Building and Environment 45(2) (2010), pp. 411-420.

Yang, H. S., Kang, J., Cheal, C. Random-Incidence Absorption and Scattering Coefficients of Vegetation. Acta Acustica united with Acustica 99(3) (2013), pp. 379-388. 